\title{
Superheavy Particle Production in High Energy Heavy Ion Collisions
}

\author{
Alexey Kurepin \\ Department of Experimental Physics, Institute for Nuclear Research, Russian Academy of Sciences, Moscow, Russia \\ Email: kurepin@inr.ru
}

How to cite this paper: Kurepin, A. (2021) Superheavy Particle Production in High Energy Heavy Ion Collisions. Journal of Modern Physics, 12, 433-439.

https://doi.org/10.4236/jmp.2021.124030

Received: February 12, 2021

Accepted: March 8, 2021

Published: March 11, 2021

Copyright $\odot 2021$ by author(s) and Scientific Research Publishing Inc. This work is licensed under the Creative Commons Attribution International License (CC BY 4.0).

http://creativecommons.org/licenses/by/4.0/

\begin{abstract}
The existence of several $\mathrm{TeV}$ superheavy particles (SHPs) is predicted by theories beyond the Standard Model. Particles with a mass exceeding the energy in the center of mass in the collision of protons with protons can be produced in subthreshold heavy ion collisions at the LHC. The purpose of the performed research was to estimate the rate of a rare process of SHPs production. It was shown that the data on the subthreshold production of antiprotons can be explained by the phenomenological parton model. The obtained parton distribution function was used to determine the number of SHPs produced in subthreshold heavy ion collisions at the LHC. In one month of collision of lead with lead, the yield of $16 \mathrm{TeV}$ particles is about 70 per year. To study the kinematically forbidden phenomena in proton-proton interactions in collisions of heavy nuclei at the LHC, an experiment on the production of antiprotons is proposed in the ALICE fixed target project.
\end{abstract}

\section{Keywords}

Particle Physics, Heavy Ion Collisions, Parton Model, Antiproton Production

\section{Introduction}

In Grand Unification Theory, it is assumed that the first massive particles which are responsible for the symmetry breaking up to the symmetry of the Standard Model at the distances larger than $10^{-29} \mathrm{~cm}$ have masses of the order of $10^{12} \mathrm{TeV}$. In the simple version of this theory next masses arise only at distances about $10^{-16} \mathrm{~cm}$, where the particles with masses of the order of $10^{2} \mathrm{GeV}$ should be considered. They are connected to the breaking of electroweak symmetry, so called Higgs scalar bosons. Therefore a rather artificial hypothesis was proposed on the existence of the "gauge dessert", i.e. no particles have masses between $0.2-10^{12}$ $\mathrm{TeV}$. However, some other possibility for high mass production is predicted by 
Large Extra Dimension theories, where our $3+1$ dimensional world is considered as a "brane" in the higher dimensional space. Then the Planck mass can be brought down to a level of the order of one TeV [1]. Then Superheavy Particles with masses of several $\mathrm{TeV}$, such as mini Black Holes, could be produced if the extra dimension is equal to seven.

Additionally, more complicated models have been proposed with multistep breaking of the symmetry at super high energy down to the symmetry of the Standard Model, where some new particles could be adopted with masses in the interval filling the "gauge dessert". One of the most interesting possibilities provides theory based on new symmetry principle connecting the particles with different statistics, so-called Supersymmetry Theory. New types of heavy quarks and leptons are introduced in this theory: s-quarks and s-leptons and corresponding supersymmetry partners. In that respect, we can take into account new possibilities for producing the masses up to $10^{3} \mathrm{TeV}$ with the LHC with heavy ions as an opportunity to search for new superheavy supersymmetry particles. We can expect rather small coupling between these new types of physical objects and particles of the ordinary world. Therefore, one can look for superheavy particles with a large lifetime. Although there are several proposals for the search for long-lived particles, none of them are considering particles with a mass of several $\mathrm{TeV}$.

The unique possibility of relativistic heavy ion collisions is production with considerable probability of particles which are kinematically forbidden for nucleon-nucleon collisions with the same energy per nucleon. The essential increase in the "subthreshold" antiproton production cross section for nucleus-nucleus collision normalized per one nucleon in comparison to nucleon-nucleus collision was observed [2] [3] [4] [5] [6]. This increase can reach two orders of magnitude and cannot be explained by Fermi motion in the incident and target nuclei [7]. However, this approach took into account only nucleon-nucleon collisions. "Subthreshold" particle production could be due to multinucleon or multiquark correlation in heavy nuclei. It can be assumed that this correlation is determined by the parton distribution function with a scaling parameter greater than unity.

The total energy in the center of mass system for $\mathrm{Pb}-\mathrm{Pb}$ collisions at the $\mathrm{LHC}$ is approximately $1150 \mathrm{TeV}$. Therefore, the unique possibility could be considered to produce Super Heavy Particles (SHPs) by nucleus-nucleus collisions with masses much larger than the center of mass energy in p-p collisions. To date, there is no theory for calculating the cross section for SHPs production in collisions of heavy ions. However, to plan a possible experiment, it is necessary to estimate the number of particles produced. In this article, it is proposed to use for this estimate the parton distribution function obtained for the subthreshold antiproton production.

\section{Parton Model for Particle Production in Heavy Ion Collisions}

For the analysis of the subthreshold hadron production at intermediate energies 
the phenomenological parton model was proposed [8]. It was observed that the subthreshold and near threshold production cross section in proton-nucleus and nucleus collisions for pions, kaons and antiprotons could be expressed as a universal function of the scaling quark-parton parameters, such as the Bjorken scaling parameter $x$ for deep inelastic electron scattering, but take into account the production of massive particles and is now different in incident $(Z)$ and in target $(X)$ nuclei. Values $Z$ and $X$ larger than 1 arise to provide the production of particles at subthreshold energies for nucleon-nucleon process. As usually a physical interpretation of these scaling parameters in a quark-parton model means that $X P_{b}$ correspond to parton 4 -momentum in a target nuclei, where $P_{b}$ is 4-momentum of a nucleon in a target nucleus. $X$ could take the values in an interval $0<X<A$, where $A$ is the atomic number of a target. On the other hand the same parton model could be applied to the incident particle $Z P_{a}$.

For antiproton production the value of $X$ for fixed $Z$ could be obtained:

$$
X=\frac{Z\left(P_{a} P_{d}\right)+Z m_{a} m_{n}+\frac{1}{2}\left(m_{n}^{2}-m_{d}^{2}\right)}{Z\left(P_{a} P_{b}\right)-Z m_{a} m_{b}-\left(P_{b} P_{d}\right)-m_{b} m_{n}}
$$

which is derived from the conservation of the 4-momentum in the collision. Here $P_{d}$ is the 4-momentum of the antiproton, $m_{a} m_{b} m_{n}$-mass of the nucleon in the incident and in target nuclei, and $m_{d}$-mass of the antiproton. Because of the exponential dependence of the production cross section on the scaling parameters, this equation matches the smallest values of $X$ and $Z$ parameters corresponding to the smallest energy in the center of mass of partons interaction.

For proton-nucleus and nucleus-nucleus interactions, the experimental data over a wide energy interval for different incident and target nuclei could be approximated with the same scaling law. Those are the data for antiproton production with $\mathrm{Ne}$ and Ni nuclei with $1-2 \mathrm{GeV} /$ nucl. at GSI [6], proton and carbon nuclei with $3.65 \mathrm{GeV} /$ nucl. at JINR [2] [3], with a deuteron beam at KEK [5] and protons and a Si-beam at LBL for antiproton and $\mathrm{K}^{-}$production [4]. The scaling in Figure 1 is observed with a $Z$-parameter equal 1 for proton beam, 1.3 for deuteron, 2 for carbon beams and 3 for $\mathrm{Si}, \mathrm{Ne}$, and $\mathrm{Ni}$ nuclei [8]. The production cross sections were reduced by $\left(A_{1} A_{2}\right)^{0.43}$ according to $A$-dependence investigated in [9] and due to the different absorption nuclear effects for pions, kaons and antiprotons.

The considerable increase in the ratio of kaon to pion production rates in nucleus-nucleus collision compared to the same ratio in deuteron-nucleus collision was also explained by the scaling dependence on the $Z$ parameter for $Z<1$, but with the parameter $X=2$ for incident carbon nuclei and $X=1.5$ for incident deuterons [10].

The considerable increase in the production rates for kaons and antiprotons compared to pions in nucleus-nucleus collisions was interpreted as the indication of collective parton effect and was quantitatively reproduced by the introduction of scaling parameters larger than one. From Figure 2 we see that the 


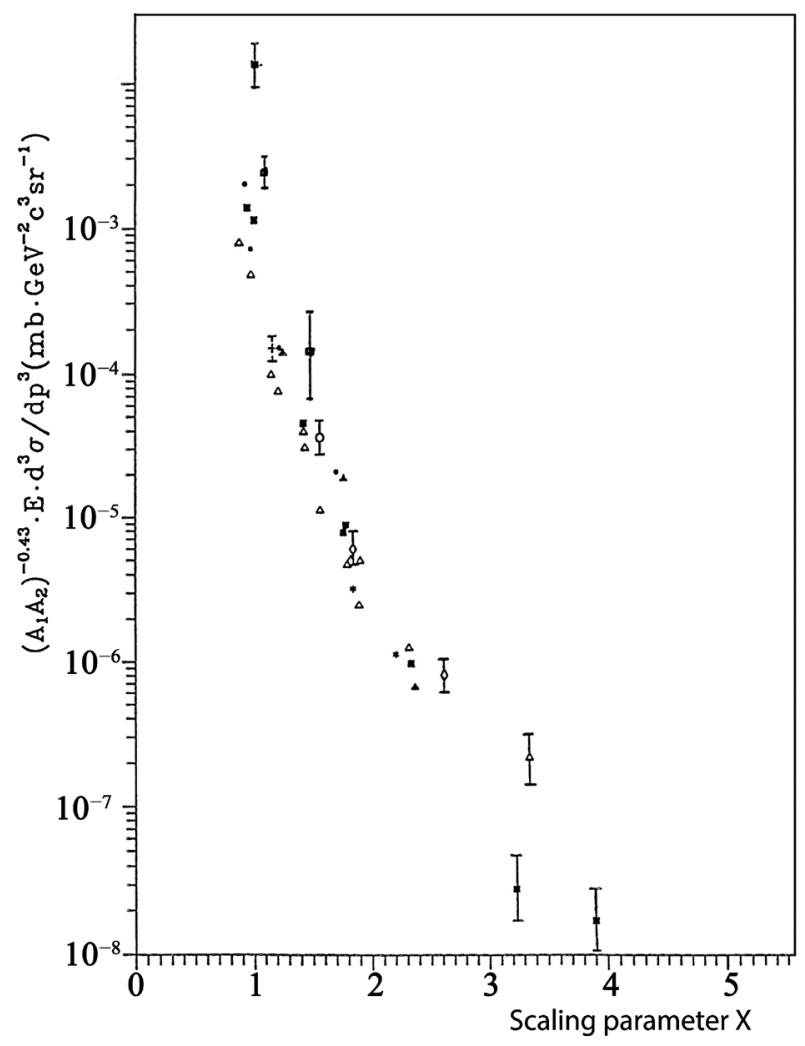

Figure 1. Systematics of Lorentz invariant antiproton subthreshold production cross section dependence on the scaling parameter $X$ with $Z=1$ for incident protons, $Z=1.3$ for deuterons, $Z=2$ for carbon ions, $Z=3$ for heavy nuclei. Points $\mathrm{p}+\mathrm{C}$, open circles d $+\mathrm{C}$, crosses $\mathrm{C}+\mathrm{C}$ and $\mathrm{C}+\mathrm{Cu}$ [2] [3], triangles $\mathrm{p}+\mathrm{C}$ and $\mathrm{d}+\mathrm{C}$ [5], squares $\mathrm{p}+\mathrm{Cu}$ [7], rhombs $\mathrm{Si}+\mathrm{Si}[4]$, stares $\mathrm{Ne}+\mathrm{Sn}$ and $\mathrm{Ni}+\mathrm{Ni}[6]$. Only some statistical errors are presented to show the order of uncertainty for the measurements of subthreshold production.

dependence of the Lorenz invariant inclusive cross section, or of the structure function, on the scaling parameter is close to the exponent. The "subthreshold" structure function in Figure 2 could be approximated as:

$$
\left(A_{1} A_{2}\right)^{-0.43} \cdot E_{1} \frac{\mathrm{d}^{3} \sigma}{\mathrm{d} p^{3}}\left[\mathrm{mb} \cdot \mathrm{GeV}^{-2} \cdot \mathrm{c}^{3} \cdot \mathrm{sr}^{-1}\right]=0.57 \exp (-X / 0.158)
$$

where $A_{1}$ and $A_{2}$ are the mass numbers of colliding nuclei.

This curve can be used to describe the experimental data in the range of cross section $10^{-1}-10^{-5} \mathrm{mb}$ with an accuracy less than an order of magnitude for $X=1$ - 3 for the data from approximately 2 to $6 \mathrm{GeV}$ per nucleon.

The enhancement of kaon and antiproton production is quantitatively reproduced by the introduction of a large $Z$ scaling parameter: $Z=1.3$ for deuterons, $Z=3$ for all heavy colliding nuclei. For incident protons $Z$ is equal to one.

This scaling was observed for different quark flavors. Therefore one can expect that the model can be applied to some heavier unknown quarks and that these collective phenomena could be due to some general space-time properties of nucleus-nucleus interaction or to the universal structure function of a nucleus. 


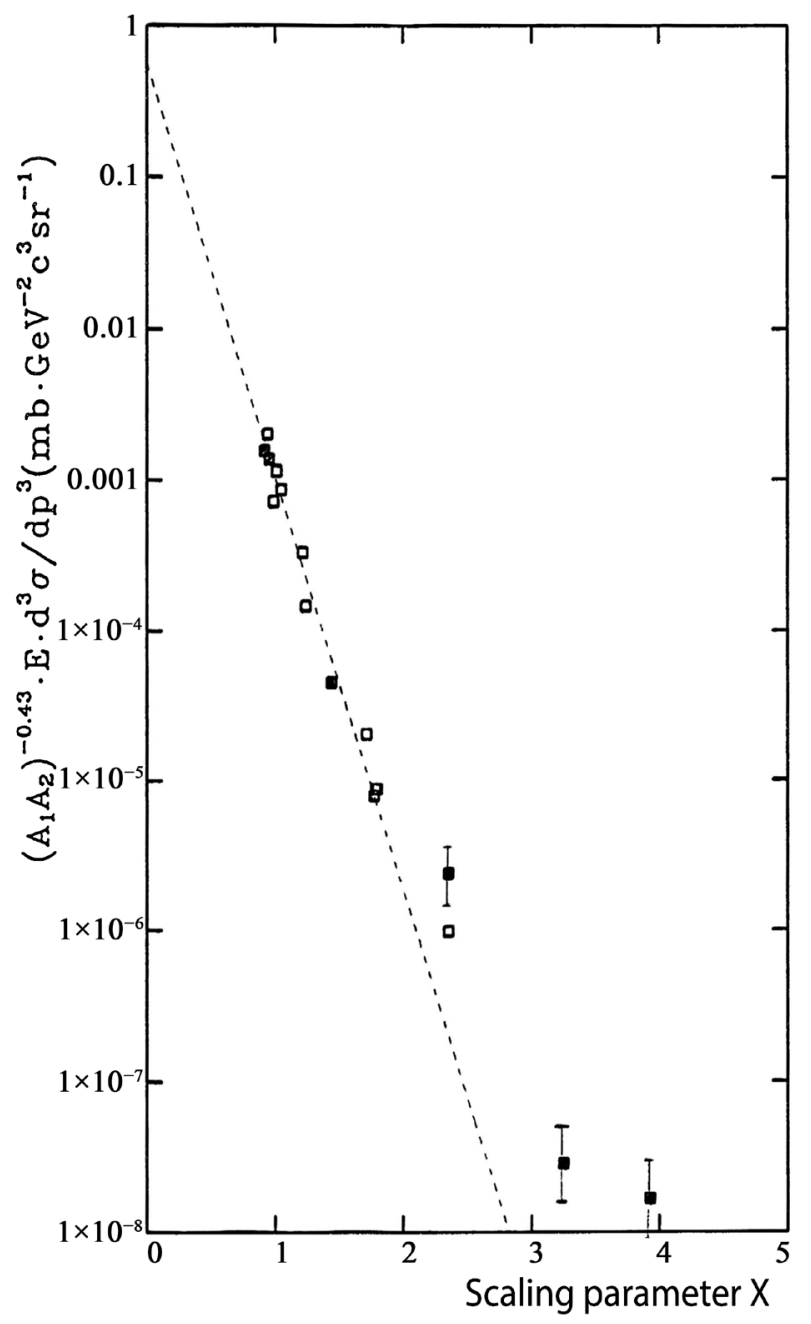

Figure 2. $\chi^{2}$-Fit to the invariant antiproton production cross section dependence on the $X$-scaling parameter. Only data for the interaction of protons with carbon (open marks) and with copper (filled marks) are shown.

\section{Estimate of Production Yield of Super Heavy Particle Production}

From Equation (2) we see that the cross section strongly depends on the parameter $X$. Therefore, to produce SHP with considerable probability, one should look for the kinematics with the smallest $X$ and $Z$ possible. To estimate the SHP production cross section for the LHC using the scaling dependence of the structure function for $M \gg m_{n}$ we can determine $Z$ and $X$ from the relation on the threshold for SHP production:

$$
\sqrt{S}=2 E \sqrt{X \cdot Z}=M
$$

where $M$ is the mass of SHP, $m_{n}$ is the mass of nucleon, $E$ is the energy per nucleon of colliding nuclei.

To estimate the production rate of SHP with the mass $M$, Equation (2) could be used with $Z=3$ for nucleus-nucleus collision and $X$ to be determined from Equation (3). 
The absolute value of the momentum $P$ of SHP is obtained from the equation

$$
X=\frac{2 Z\left(E \cdot E_{1}-E \cdot P \cdot \cos (\theta)\right)-M^{2}}{4 Z \cdot E^{2}-2\left(E \cdot E_{1}+E \cdot P \cdot \cos (\theta)\right)}
$$

which is derived from the conservation of the 4-momentum in the collision. Here $E_{1}$ is the total energy of SHP, $\theta$ is the angle between SHP and $Z$ parton.

Assuming that SHPs are produced with small transverse momentum in the narrow forward-backward cone we obtain for the masses $M$ larger than $1 \mathrm{TeV}$, the velocities of SHP are of the order of $0.1-0.9 \mathrm{c}$.

Accepting that the multi quark correlations are the intrinsic property of nuclei and do not depend on the collision energy, we can try to use the "subthreshold" structure function from Equation (2) to estimate the production rate of SHPs depending on their mass in $\mathrm{Pb}-\mathrm{Pb}$ collisions:

$$
E_{1} \frac{\mathrm{d}^{3} \sigma}{\mathrm{d} p^{3}}\left[\mathrm{mb} \cdot \mathrm{GeV}^{-2} \cdot \mathrm{c}^{3} \cdot \mathrm{sr}^{-1}\right]=56 \exp (-X / 0.158)
$$

For the obtained luminosity $L=2 \times 10^{27} \mathrm{~cm}^{-2} \cdot \mathrm{sec}^{-1}$ for one month of the run and an opening angle of approximately $100 \mathrm{msr}$ for the forward detector we obtain an estimate of the SHP yield for a mass approximately $16 \mathrm{TeV}$ near the threshold on the order of 70 per year. For larger SHP masses the production cross section is greatly reduced due to the increasing parameter $X$ in the exponential dependence. The results obtained can be used when planning an experiment at the LHC. Assuming SHPs are long-lived particles, the forward detectors will see very large signals. For a short lifetime, barrel detectors will detect events with anomalously high multiplicity.

The question remains about the possibility of applicability of the empirical scaling law, obtained at intermediate energies, to interactions at ultrahigh LHC energies. Unfortunately, it is impossible to measure the scattering and production of particles kinematically forbidden for nucleon-nucleon interactions at angles greater than 90 degrees on collider experimental facilities, that is, at $X>1$.

Very promising possibilities for studying subthreshold phenomena are opened up in the case of experiments with a fixed target at the LHC [11] [12] [13]. The AFTER or ALICE-FT project provides for the placement of a solid or gaseous target in the beam halo when using all detectors of the ALICE facility. In this case, it is possible to measure the production of particles, in particular antiprotons, at large parameters $X$ [13] even at $X>1$, which will make it possible to check the existence of the scaling, considered in this work, at the energies of ion beams at the LHC. For a fixed target with a beam energy of $2.76 \mathrm{TeV}$ per nucleon and $Z=3$ from Equation (4) we obtain $X=1.75$ for a production angle of 28 degrees.

\section{Conclusion}

The extrapolation of the scaling observed in the parton model for subthreshold antiproton production in the collision of heavy ions to high energies was per- 
formed, in order to obtain estimates of the cross section and yield for the production of particles with masses up to $16 \mathrm{TeV}$. It is proposed to investigate subthreshold phenomena in an experiment with a fixed target at the LHC.

\section{Acknowledgements}

This research was supported by the RFBR/CNRS grant 18-52-15007.

\section{Conflicts of Interest}

The author declares no conflicts of interest regarding the publication of this paper.

\section{References}

[1] Rubakov, V.A. (2003) Physics-Uspekhi, 46, 211-218. https://doi.org/10.1070/PU2003v046n02ABEH001355

[2] Baldin, A.A., et al. (1988) JETP Letters, 48, 137-140.

[3] Baldin, A.A., et al. (1990) Nuclear Physics A, 519, 407-411. https://doi.org/10.1016/0375-9474(90)90644-2

[4] Carroll, J.B., et al. (1989) Physical Review Letters, 62, 1829-1832. https://doi.org/10.1103/PhysRevLett.62.1829

[5] Chiba, J., et al. (1993) Nuclear Physics A, 553, 771-774. https://doi.org/10.1016/0375-9474(93)90696-U

[6] Schroeter, A., et al. (1993) Nuclear Physics A, 553, 775-778. https://doi.org/10.1016/0375-9474(93)90697-V

[7] Shor, A., Perez-Mendez, V. and Ganezer, K. (1990) Nuclear Physics A, 514, 717-733. https://doi.org/10.1016/0375-9474(90)90019-I

[8] Kurepin, A.B., Shileev, K.A. and Topilskaya, N.S. (1997) Genshiryoku Kenkyu, Tokyo, 41, 177-182.

[9] Baldin, A.A., et al. (1995) Nuovo Cimento A, 108, 139-146.

[10] Kurepin, A.B., Shileev, K.A. and Topilskaya, N.S. (1996) Acta Physica Polonica B, 27, 3077-3080.

[11] Kurepin, A.B., Topilskaya, N.S. and Golubeva, M.B. (2011) Physics of Atomic Nuclei, 74, 446-452. https://doi.org/10.1134/S1063778811030124

[12] Brodsky, S.J., Fleuret, F., Hadjidakis, C. and Lansberg, J.P. (2013) Physics Reports, 522, 239-255. https://doi.org/10.1016/j.physrep.2012.10.001

[13] Kusina, A., et al. (2019) Probing the High-X Content of the Nuclei in the Fixed-Target Mode at the LHC. arXiv:1901.07950 [hep-ex] https://doi.org/10.22323/1.345.0110 